\title{
Verifying Software Requirements with XSLT
}

\author{
Amador Durán, Antonio Ruiz, Beatriz Bernárdez and Miguel Toro \\ Department of Computer Languages and Systems \\ University of Seville, Spain \\ e-mail: \{amador,aruiz,beat,mtoro\}@lsi.us.es
}

\begin{abstract}
In this article, we present an approach for the automatic verification of software requirements documents. This approach is based on the representation of software requirements in $X M L$ and the usage of the XSLT language not only to automatically generate requirements documents, but also to verify some desired quality properties and to compute some metrics. These ideas have been implemented in REM, an experimental requirements management tool that is also described in this paper.
\end{abstract}

Keywords: requirements engineering, requirements verifcation, XML, XSLT

\section{Introduction}

Paraphrasing Boehm [5], requirements validation and verification can be informally defined by the questions " $A m$ $I$ building the right requirements?" (validation) and "Am I building the requirements right?" (verification).

In other words, the goal of requirements validation is to ensure that requirements documents contain actual requirements and that these requirements are all the known requirements by the time the requirements documents are baselined.

On the other hand, the goal of requirements verification is to ensure the quality of requirements according to desired quality properties. Some of these quality properties have to do with requirements semantics but others have to do with syntactic, structural or pragmatic aspects of requirements (see [12] for a complete classification of quality properties of requirements).

Verification of semantic properties of requirements is closely related to requirements validation ${ }^{\mathbf{1}}$ and requires human participation, whereas verification of non-semantic properties should be as automated as possible.

In this article, we present an automated approach for the verification of some quality properties of requirements. Most of these properties can be classified as non-semantic, but we have also developed some heuristics to check potential problems with some semantic properties. Our approach is based on the emergent technology built around XML [4] and its companion language XSLT [3].

The rest of the article is organized as follows. First, we briefly describe the basics of XML and XSLT needed to understand the following sections. Then, we describe REM, an experimental requirements management tool $[8,9]$, the

\footnotetext{
${ }^{1}$ Distinction between requirements verification and validation is sometimes subtle and many authors use both terms interchangeably.
}

XML model of requirements used by REM and how XSLT can be used to verify some quality properties of requirements expressed in XML. Finally, we discuss some related work, present some results and point out future work.

\section{XML and XSLT}

\section{XML Basics}

There are millions of web pages written in HTML available in Internet. In these web pages, pure information is mixed with formatting elements, making the automatic processing of information very difficult. XML $[4]$ is a language designed for representing pure information in Internet. Information in XML is represented by elements. An XML element is made up of a start tag, an end tag, and other tags or data in between. For example, for representing the information about a book, we might have the following XML element named book:

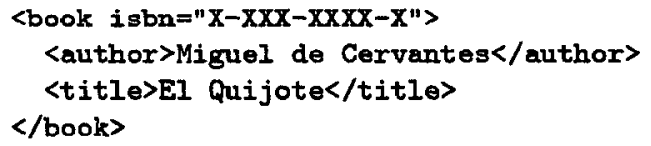

As you can see, the information about a book is between the jbook $_{i}$ and $i /$ book $_{i}$ tags and it is easy to parse by a computer program. The author and title elements are considered as children of the book element, thus forming a hierarchy. An XML document must always have one and only one root element at the top of its hierarchy.

In order to allow information interchange between two or more parties using XML, they must agree about element grammar and semantics. Element grammar is specified as regular expressions in DTDs (Document Type Definitions) [4]. For example, the DTD fragment for the previous XML data would be the following:

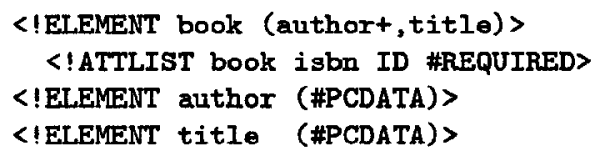

where it is stated that a book element can contain one o more author elements and only one title element. An XML element can also have attributes. For example, isbn is defined as a required identification attribute of book, i.e. there cannot exist two books with the same value for the isbn attribute in the same XML document. Those elements that contain only text are said to contain \#PCDATA, that stands for parsed character data. 


\section{Transforming XML}

There are many situations in which XML data need to be transformed. For example, for presenting XML data as an HTML page. XSLT [3] is a language based on transformation patterns. An XSLT stylesheet, which is also a an XML document, searches for patterns in the XML data and applies programmed transformations, thus generating some output results. For example, if we wanted to show information about books in a web browser, we could apply the following XSLT transformation rule:

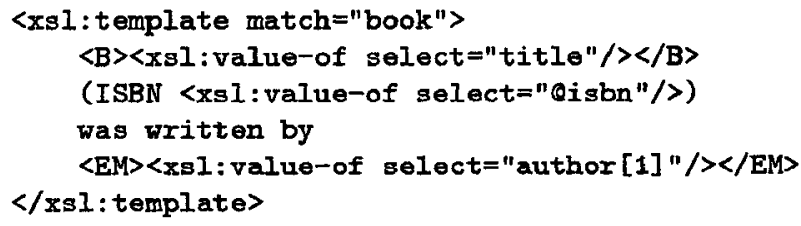

The informal semantics of this XSLT rule are "when you find a book element, generate its title in boldface, then its ISBN attribute (notice the @ prefix for attributes), and then its first author in emphasized mode". In the XSLT code, text literals like HTML tags can be mixed with element values, which are obtained by means of the xsl:value-of statement. If we applied this XSLT rule to the previous XML data, the result of the transformation would be something like this when rendered in a web browser:

El Quijote (ISBN X-XXX-XXXX-X) was written by Miguel de Cervantes

Although there are many more details about XML and XSLT, we think that this brief introduction should be enough for those readers not familiar with XML technologies in order to understand the rest of this article.

\section{REM: An XML-based Requirements Management Tool}

REM (REquirements Manager) is an experimental requirements management tool developed by one of the authors $[8,9]$. In REM, a requirements engineering (RE) project is considered to be composed of three documents:

1. a customer-oriented requirements document (the requirements document [13]), usually containing requirements in natural language expressed in terms of customer's vocabulary, also known as $C$-requirements [7].

2. a developer-oriented requirements document (the specification document [13]), usually containing requirements models and more technical information, also called $D$ requirements $[7]$.

3. a registry for detected conflicts and negotiation support.

In REM, C-requirements and conflicts are expressed in natural language using predefined requirements templates and some linguistic patterns (see [9] for details). For expressing $\mathrm{D}$-requirements, we have chosen a subset of the UML [6]

\section{REM Architecture}

REM documents, i.e. RE projects composed of the three documents previously described, are stored in relational lightweight databases. When the user creates a new REM document, the basic structure is taken from a REM base document (see figure 1), that can be empty or can contain the mandatory sections of software requirements standards like [1] or [15]. Any ordinary REM document can be selected as a base document, so users can create their own base documents or reuse other REM documents.

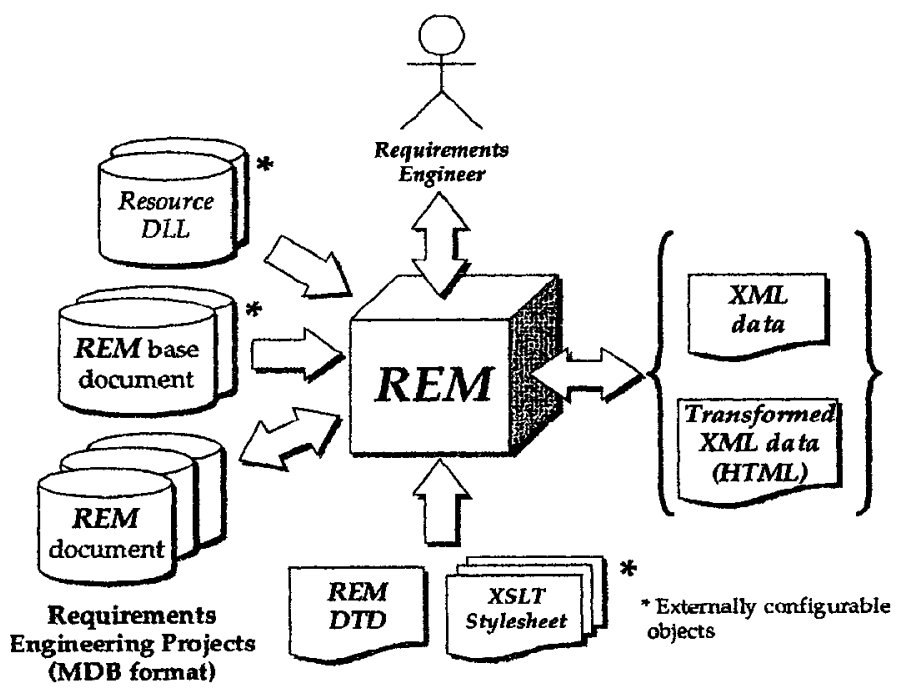

Figure 1: REM Architecture

In order to provide immediate feedback on user actions, REM generates XML data corresponding to the document being edited, applies an external XSLT stylesheet that transforms XML data into HTML and shows the resulting HTML to the user. In this way, whenever the user changes a requirements document, he or she can see the effects immediately.

In a similar way the REM base document can be tailored, the user can also change document appearance by selecting or creating different external XSTL stylesheets. The default XSLT stylesheet generates a highly hyperlinked document, easing navigation of requirements documents (see right side of figure 2).

Other configurable aspect of REM is the language of the user interface. The user can choose it by selecting an external resource dynamic link library (DLL). At the moment of writing, we have developed two external resource DLLs for REM, one in Spanish and other in English. Another one in Portuguese is under development.

\section{REM User Interface}

The user interface of REM presents two different views to the user (see figure 2). On the left, the user can see a tabbed view with three tree views, one for each requirements document in the RE project. On the right hand, the result of the XSLT 


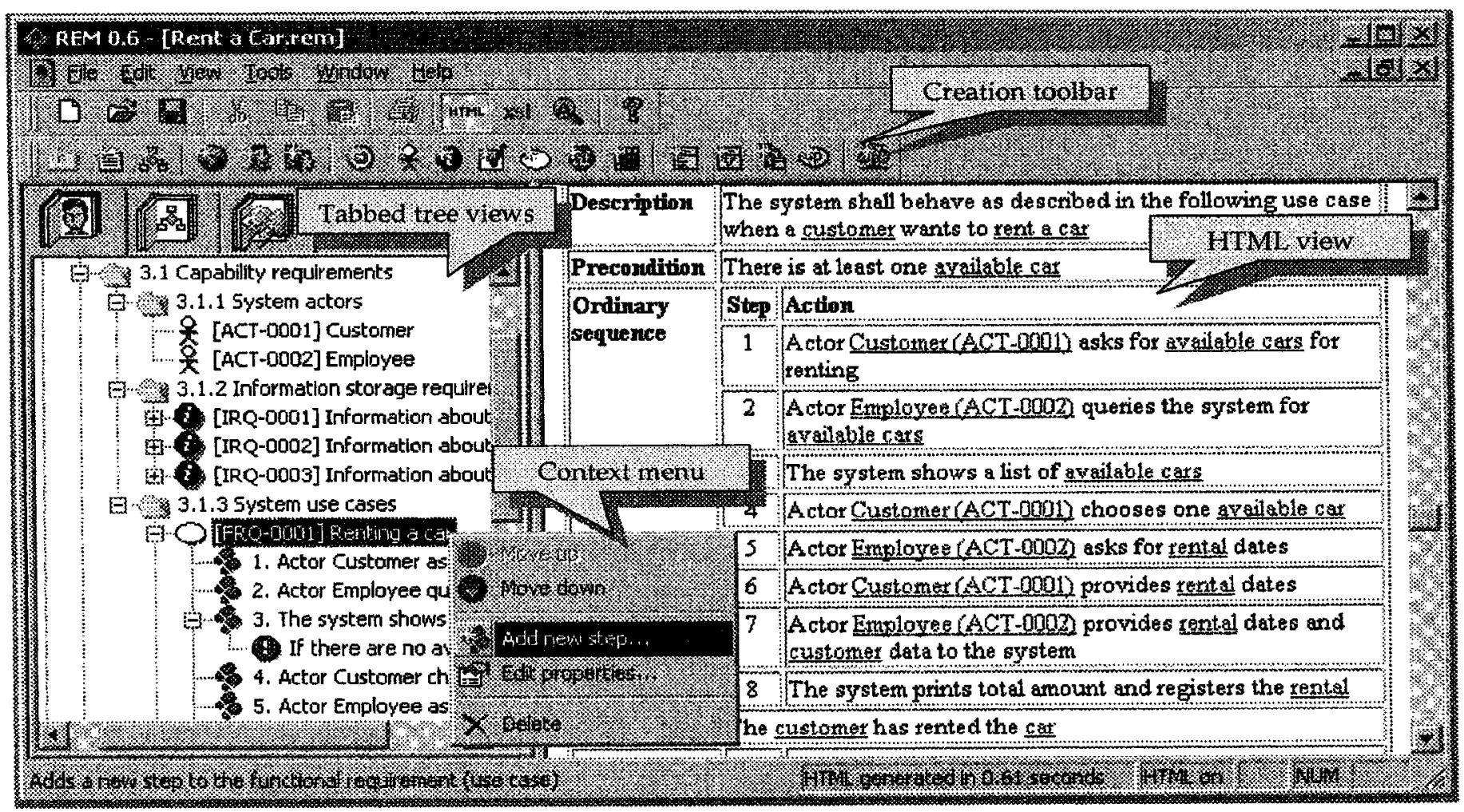

Figure 2: REM User Interface

transformation of the XML data is presented to the user in a embedded web browser.

In any of the three tree views, the user can directly manipulate objects by drag and drop or by context menus. Only actions that have sense can be performed, following a correctby-construction approach, thus increasing quality and saving verification effort.

For example, actions of use case steps can be of three different classes (see figure 4): actor action, if the action is performed by an actor; system action if the action is performed by the system, or use case action, if the action consists of performing other use case, i.e. an use case inclusion or extension [6]. Actor actions and use case actions can be created only if some actor or some use case have been previously created. In general, objects can be created by means of context menus on potential parents or by means of the creation toolbar.

\section{XML Model of Requirements in REM}

REM is based on an UML [6] model of requirements (a partial view of this model is shown in figure 4). The main object class of the model is the Requirements Document, that is composed of a sequence of REM objects. See figure 3 for a classification of REM objects.

We have translated our UML model of requirements into a relational schema and into a DTD. As an example, the Use-
Case class in figure 4 has been translated into the following DTD element definition:

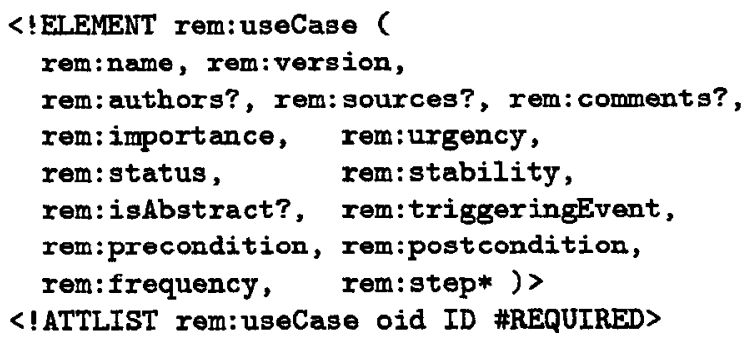

Many of the elements in the previous DTD fragment (comments, triggeringEevent, pre and postcondition), contains only text, i.e. natural language. In REM, text can be composed of any combination of free text, references to other objects and TBD (To Be Determined) marks, defined as follows:

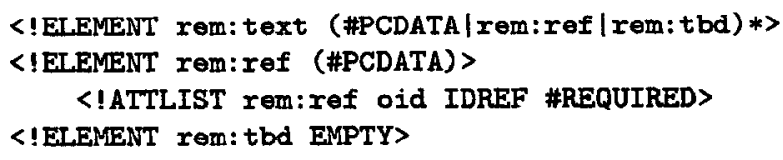

where the rem:ref element must have a required attribute called oid that it is declared as an IDREF, i.e. a reference to other element with a matching identification attribute value. An IDREF attribute is very similar to a foreign key in relational databases. 


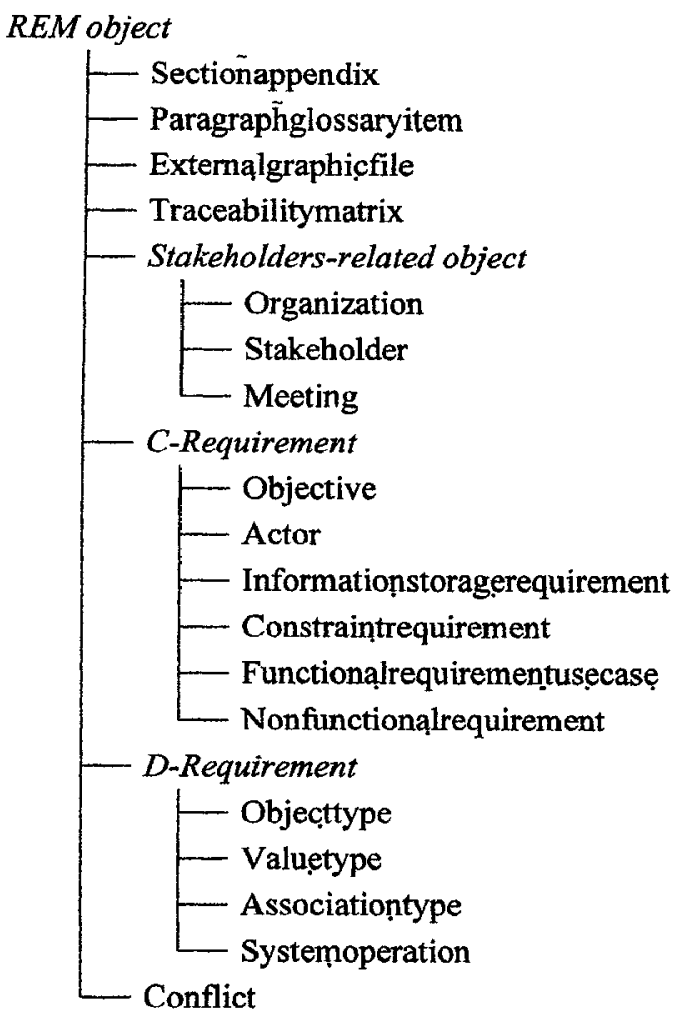

Figure 3: Classification of objects in REM

The rem:tbd element is declared as an EMPTY element, i.e. it cannot have neither subordinate elements nor data. It is simply a mark.

\section{Using XSLT as a Requirements Veri- fication Language}

In the following sections we describe how some of the quality factors described in [10] can be automatically verified using XSLT when requirements are electronically stored in XML format according to the REM DTD.

\section{Unambiguity}

A requirement is unambiguous if and only if has only one possible interpretation [1]. Obviously, this is a semantic property of a requirement and cannot be automatically verified, but we can give some hints about potential ambiguities in a requirements document.

We agree with Leite [11] in the importance of understanding the language of the problem and in the importance of building a glossary (called Language Extended Lexicon, LEL, in [11]). Following Leite, the glossary should follow two principles: the principle of circularity, (the glossary must be as self-contained as possible) and the principle of minimal vocabulary (use as much glossary items as possible in

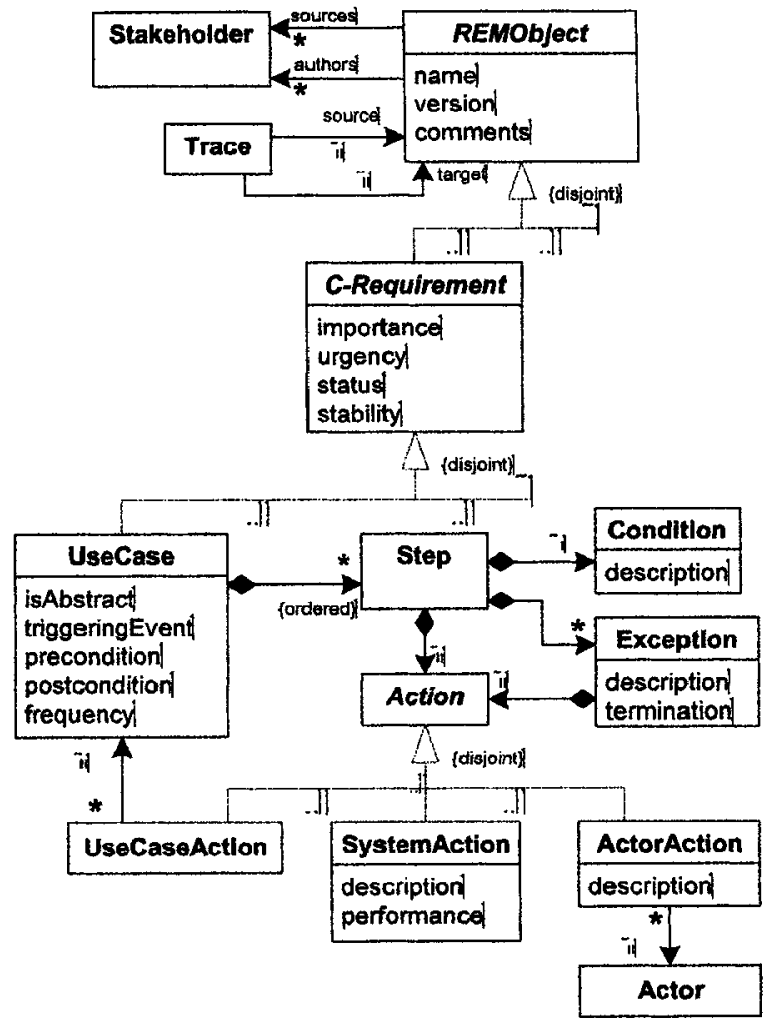

Figure 4: UML model of use cases in REM

your requirements descriptions). Leite's principles cannot guarantee unambiguity, but they can help to build unambiguous, understandable, verifiable, consistent, concise, and cross-referenced requirements [10].

XSLT can be used to measure glossary circularity (GLC) and minimality of vocabulary (MOV). GLC can be measured as the ratio between glossary items and references to glossary items from other glossary items. The following XSLT code, where we have declared a variable for the sake of readability, can be used for computing GLC:

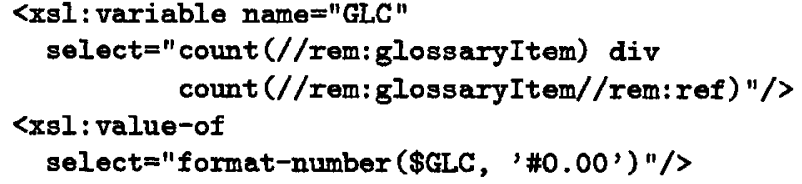

where the expression //rem:glossaryltem is an XPath expression [2] meaning "any rem:glossaryltem element descendant of the root", whereas the expression //rem:glossaryltem//rem:ref means "any rem:ref element descendant of any rem:glossary/tem descendant of the root". In XPath, the language for building navigation expressions over XML trees, an element is considered as descendant of other element if it is its child at any level of depth in the hierarchy.

A similar ratio between the number of references to glossary items in requirements and the number of requirements can be used to measure MOV. From the MOV viewpoint, 
it is also possible to detect those "suspicious" requirements that do not have any reference to any glossary item in their text. Since those requirements are not using the vocabulary of the customer, they should be checked for potential problems of ambiguity or understandability [10]. For example, if we want to know which use cases are "suspicious", we can use the following XSLT code:

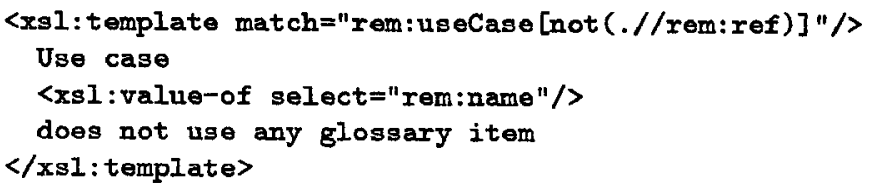

where the match expression uses brackets to select only those use cases with no descendant references. Another possibility is to determine a threshold value for the number of references per requirement and consider as suspicious all requirements with a number of references under the threshold. In that case, the match expression would be rem:useCase[count(.//rem:ref) ; $m$ ], with $m$ being the MOV threshold.

\section{Completeness}

A requirements document is complete if it includes [10]:

1. Everything that the software is supposed to do, i.e. all the requirements

2. Responses of the software to all classes of input data in all realizable situations

3. Page numbers, figure and table names and references, a glossary, units of measure and referenced material

4. No sections marked as TBD

In our approach, the third completeness condition is partially satisfied by means of the correct-by-construction paradigm of REM: figure and table names are automatically generated, references are automatically inserted and updated, and the user can easily create a glossary. If we want to be sure about the existence of a section named Glossary, we can apply the following XSLT code:

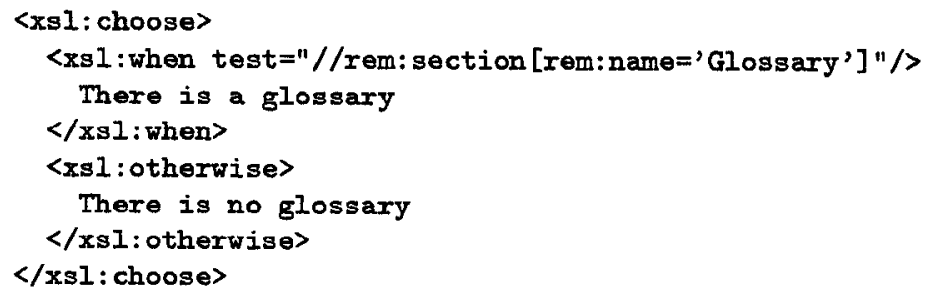

where the structure formed by xsl:choose, xsl:when and xsl:otherwise is basically an if-else-endif statement with multiple else branches. Notice that if we want to check the existence of an element we cannot use an XSLT template. If there is no such an element, the template will never match and we will have no output.
Similar XSLT code can be used to verify if requirements documents are organized [10], i.e. if they have mandatory sections in the mandatory order with mandatory content.

The fourth condition of completeness, the absence of TBD marks, can be easily verified using XSLT. If we want to know how many TBD marks are in a requirements document we can apply the following XLST code:

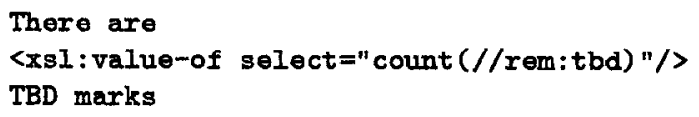

that would generate in the output the number of occurrences of elements of type rem:tbd anywhere in the XML data. If we want to be more precise and we want to know what use cases have TBD marks inside their text and how many TBD marks they have, we could write the following XSLT code:

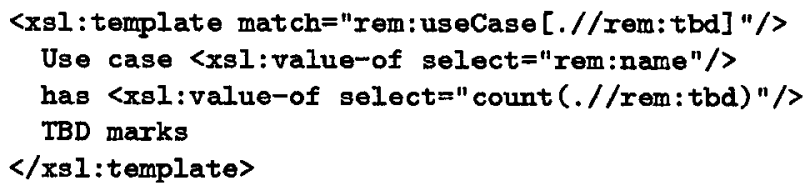

in which the select expression "remiuseCase[.//rem:tbd]" means "any use case with at least one descendant of type rem:tbd".

\section{Traceability}

In [10], a requirements document is said to be traceable if and only if it is written in a manner that eases the referencing of each individual requirement. Since REM assigns automatically an unique identifier to every requirement (the required identifier attribute oid, see the DTD for use cases), this quality factor does not have to be verified explicitly.

What it must be checked is if the origin of every requirement is clear, i.e. if requirements are traced [10]. In our UML model of requirements, any REM object can be traced to and from other REM objects and to their human sources and authors (see figure 4). Checking if a requirement has sources and authors and if it is traced to or from other requirements is easy with XSLT. For example, the following XSLT template will match all use cases with no human sources:

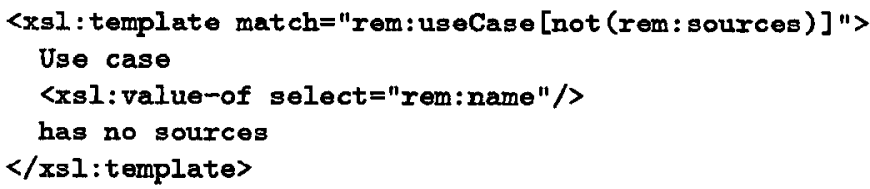

And this XSLT template will match all non functional requirements not traced to other REM objects:

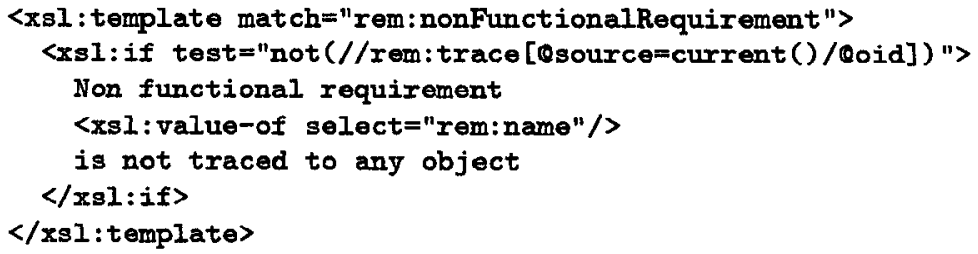


In REM, traces are defined as elements with two required attributes of type IDREF, namely source and target. The user of REM can also use traceability matrices for visual checking of non-traced requirements.

\section{Other verifiable quality factors}

Applying the same ideas, other quality factors defined in [10] can be verified using XSLT, for example:

- What requirements are not annotated with relative importance, relative stability or version.

- What requirements have potentially ambiguous words in their description, like easy to, user-friendly, etc. by means of XSLT string functions like contains [3].

- If use cases are not well structured, i.e. if there are too few or too many includes or extends relationships.

- What use cases have too few or too many steps, or too much exceptions, i.e. too many alternative courses.

- What defined actors do not participate en any use case.

\section{Related Work}

Most work on automated requirements verification is based on Natural Language Processsing (NLP), like [14] or [12]. Those approaches, focused on semantic analysis of requirements, usually make requirements engineers write requirements in a subset of natural language, demand many computer resources and have not been widely adopted in industry.

The Automated Requirement Measurement (ARM) tool $[16]$, is probably the most related work to the approach presented in this article. It is a simple yet powerful tool that scans requirements documents searching for indicators, i.e. words that have been identified as indicators of good or bad quality properties.

Our approach does not use NLP but an open, simpler and lighter technology like XML/XSLT. We can offer the same functionality of ARM plus all additional verification described in this paper, and the user of REM can defined his or her own XSLT verification stylesheets. From a practical point of view, we think that our results are useful for the average requirements engineer.

\section{Conclusions and Future Work}

In this article we have briefly presented an automated approach for the verification of software requirements. Our approach is based on a open technology like XML and XSLT. In fact, if requirements are represented in XML using a different DTD, many of the XSLT code presented in this paper should be easily adapted. Our approach does not need hard computer resources and it has proved to be useful when used with our students at the University of Seville.
Our future work is focused in developing quality metrics, so we can detect potential problems with requirements comparing quantitative values. We expect to identify some useful metrics soon by applying data mining techniques to the requirements documents generated by our students.

Acknowledgments This work is partially funded by the Spanish CICYT project GEOZOCO TIC 2000-1106-C0201 and by the international CYTED project WEST.

\section{References}

[1] IEEE Recommended Practice for Software Requirements Specifications. IEEE/ANSI Standard 830-1998, Institute of Electrical and Electronics Engineers, 1998.

[2] XML Path Language (XPath) 1.0. W3C Recommendation, November 1999.

[3] XSL Transformations (XSLT) 1.0. W3C Recommendation, November 1999.

[4] Extensible Markup Language (XML) 1.0 (Second Edition). W3C Recommendation, October 2000.

[5] B. W. Boehm. Verifying and Validating Software Requirements and Design Specifications. IEEE Software, 1(1):75-88, 1984.

[6] G. Booch, J. Rumbaugh, and I. Jacobson. The Unified Modeling Language User Guide. Addison-Wesley, 1999.

[7] J. W. Brackett. Software Requirements. Curriculum Module SEI-CM-19-1.2, Software Engineering Institute, 1990.

[8] A. Durán. A Methodological Framework for Requirements Engineering of Information Systems (in Spanish). $\mathrm{PhD}$ thesis, University of Seville, 2000.

[9] A. Durán, B. Bernárdez, A. Ruiz, and M. Toro. A Requirements Elicitation Approach Based in Templates and Patterns. In WER'99 Proceedings, Buenos Aires, 1999.

[10] A. Davis et al. Identifying and Measuring Quality in a Software Requirements Specification. In Proceedings of the 1st International Software Metrics Symposium, 1993.

[11] J. C. S. P. Leite et al. Enhancing a Requirements Baseline with Scenarios. In Proceedings of the 3rd IEEE International Symposium on Requirements Engineering (RE'g7), 1997.

[12] F. Fabbrini, M. Fusani, V. Gervasi, S. Gnesi, and S. Ruggieri. Achieving Quality in Natural Language Requirements. In Proceedings of the 11 th International Software Quality Week, 1998.

[13] B. L. Kovitz. Practical Software Requirements: A Manual of Content $\mathcal{E}$ Style. Manning, 1998.

[14] N. A. Maiden, M. Cisse, H. Perez, and D. Manuel. CREWS Validation Frames: Patterns for Validating Systems Requirements. In Fourth International Workshop on Requirements Engineering: Foundation for Software Quality (RESFQ), 1998.

[15] C. Mazza, J. Fairclough, B. Melton, D. de Pablo, A. Scheffer, and R. Stevens. Software Engineering Standards. PrenticeHall, 1994.

[16] L. Rosenberg, T. Hammer, and J. Shaw. Software Metrics and Reliability. In 9th International Symposium on Software Reliability Engineering, 1998. 